\title{
An Evaluation of "Ribolola" - A Household Tuberculosis Contact Tracing Programme in North West Province, South Africa
}

Dilraj Thind ${ }^{1}$, Salome Charalambous ${ }^{2}$, Adams Tongman $^{2}$, Gavin Churchyard ${ }^{2}$, Alison D Grant ${ }^{1}$

1. London School of Hygiene and Tropical Medicine, UK

2. Aurum Institute for Health Research, Johannesburg, South Africa 
TB/HIV contact tracing in South Africa

\section{SUMMARY}

\section{Setting}

Rural/periurban community, South Africa.

\section{Objectives}

To examine the yield of tuberculosis (TB) cases, TB preventive therapy (TBPT) initiation and human immunodeficiency virus (HIV) diagnoses from household TB contact tracing.

\section{Design}

Retrospective programme analysis.

\section{Methods}

Households of index TB cases were visited and their contacts screened for TB and HIV. TB suspects provided sputum or were referred for assessment. Contacts aged $<5$ years were referred for assessment for TBPT initiation.

\section{Results}

There were 732 index TB cases (67.1\% HIV positive). Among 3627 household contacts, 3573 (98.5\%) had known outcomes, of which 183 (5.0\%) were already on appropriate treatment. Among 3390 remaining contacts, $361(10.6 \%)$ were $<5$, of whom 34 (9.4\%) started anti-tuberculosis treatment and 286 (79.2\%) started TBPT. Among 3029 contacts aged $\geq 5$ years, 93 (3.1\%) started antituberculosis treatment: 19 (20.4\%) were smear positive and $71(76.3 \%)$ were culture positive. Among contacts aged $\geq 14$ years, 794/2133 (37.2\%) underwent HIV testing, of whom 208/794 (26.2\%) tested positive.

\section{Conclusions}

Household active case finding in high TB and HIV prevalence setting obtained high yields of TB, particularly in those aged $<5$ years, and facilitated assessment for TBPT. There was a good yield of new HIV diagnoses, and a gain in efficiency due to integration within one programme.

Keywords: tuberculosis; case finding; HIV; South Africa 


\section{INTRODUCTION}

South Africa has a very high burden of human immunodeficiency virus (HIV) related tuberculosis (TB), with incidence of all forms of TB estimated at 981/100,000 in 2009 and HIV prevalence among incident cases of $60 \% .{ }^{1}$ The DOTS strategy is the mainstay of TB control, but has not contained TB incidence in settings of high HIV prevalence. ${ }^{23}$

'Active case finding' (ACF) refers to seeking out TB cases, such as by contact tracing, whereas in 'passive case finding' individuals are left to self-present to healthcare facilities. ${ }^{4}$ Household contacts of smear-positive TB cases, particularly children aged $<5$ years, are at particularly high risk for developing TB disease..$^{5-7}$ Many resource-constrained countries have policies recommending household contact tracing for TB, but such policies are often not prioritised. However, with increasing recognition of the need to identify people with HIV at an earlier stage, there could be a gain in efficiency from programmes seeking to identify both undiagnosed TB and HIV infection in settings where the two diseases are closely associated. Such means could also identify at-risk children who are often not brought to health facilities for assessment and treatment.

This paper describes the experience from a programme offering screening for active TB and for HIV among households of TB patients in South Africa.

\section{METHODS}

\section{Setting and Programme}

The 'Ribolola' ("digging deeper" in seSotho) programme, established by the Aurum Institute in January 2009 is a household TB contact tracing programme in the Rustenburg sub-district in North West Province, South Africa. The primary aim of the programme was to determine the feasibility of TB household contact tracing in this setting.

\section{Study Population and Selection}

Index TB cases were recruited from eight municipal clinics. Cases aged $\geq 12$ years and over were eligible if sputum was smear-positive. Cases aged $<12$ were eligible if treated for any form of TB, where contact tracing aimed to identify a possible infection source. Index cases were excluded if they lived alone or had been on treatment for $>3$ months (unless still sputum smear-positive after 2 months of the intensive phase), to prioritise households most recently at risk of transmission. Index cases agreeing to take part gave details of demographics and medical history. Those not known to be 
HIV positive were offered testing and counselling. Households were visited by programme staff. Standardised educational material was used to discuss TB and HIV, and household members who agreed to take part were screened by programme staff at home.

\section{Programme Procedure}

Screened household contact demographics and medical history were recorded. An eight point TB symptom screen questionnaire was conducted and, in line with national guidelines at the time, those with any of: cough for $\geq 2$ weeks; sputum production, fever, haemoptysis, dyspnoea or pleuritic chest pain within the last month; weight loss within the last 6 months; or night sweats; were considered TB suspects. Contacts aged $<12$ years were assessed for additional paediatric features, such as failure to thrive or growth retardation. Any such feature for any duration (in the absence of an obvious alternative cause) in those aged $<12$ years constituted being a TB suspect.

TB suspects with a sufficiently productive cough were educated on providing sputum samples (the first spot was sent for smear only, while the two early morning specimens were sent for smear and culture, respectively). Anyone with a positive smear and/or culture result was referred to start treatment. TB suspects were referred to government health care facilities if they were sick or unable to provide sputum. Irrespective of symptoms, contacts aged $<5$ years were referred for exclusion of active TB and TB preventive therapy (TBPT), according to South African national guidelines. ${ }^{8}$ As they could still potentially be TB cases, those aged $\geq 5$ years who were already on TBPT were nonetheless screened, and were not excluded from the subsequent analysis.

Referral attendance was followed up by programme staff, and referral outcomes were ascertained retrospectively. Contacts were regarded as 'not traced' if no outcome data could be obtained from clinics or from the individuals themselves. HIV counselling and rapid testing (finger-prick blood spot) was offered to contacts aged $\geq 14$ years who self-reported their HIV status to be negative. Contacts aged $<14$ years were eligible if their mother was HIV-positive. Any contact testing HIV-positive was offered appropriate referral.

\section{Definitions}

A household was defined as: a group of individuals who generally sleep and eat together. A TB case was defined as a contact that was sputum smear- and/or culture-positive, or who started treatment following referral and assessment. For the purpose of this analysis, contacts were defined as HIV- 
positive if they had a test performed by the programme with a positive result or, if not tested, selfreported HIV positive status.

\section{Statistical Analysis}

This evaluation aimed to describe the yield of TB and HIV cases identified by the programme. Data were entered in an Access database (Microsoft Corp, Redmond, WA, USA). Anonymised data sets were cleaned and analysed using Stata, version 11 (StataCorp, College Station, TX, USA). Among household contacts, univariable and multivariable adjusted odds ratios (ORs) with $95 \%$ confidence intervals (Cls) were used to explore variables associated with being found to be a TB case.

\section{Ethical Considerations}

Permission for the programme to recruit index patients from health facilities and refer back from the community was obtained from the Bojanala district and Rustenburg sub-district departments of health. Approval to undertake retrospective analysis of programme data was obtained from the Research Ethics Committees of the University of Witwatersrand, Johannesburg, South Africa and the London School of Hygiene \& Tropical Medicine, London, UK.

\section{RESULTS}

\section{Index Patients}

A total of 732 index cases recruited between January 2009 and July 2010; 373 (51\%) were males, and the median age was 34 (range 0-93) years, which was higher for males than females (36 years, range $0-83$ vs. 32 years, range 0-93; $P<0.001$, Mann-Whitney U-test).

HIV status was recorded for 480 index cases, of whom 322 (67.1\%) were HIV-positive. A higher proportion of females than males were HIV-positive (176/241, 73.0\% vs. 146/239, 61.1\%; $P=0.005, \chi^{2}$ test). Among those self-reporting HIV-positive status at the time of presentation to the clinic, 99/295 (31\%) reported being on antiretroviral therapy (ART).

\section{Screened Household Contacts}

A total of 3627 contacts were screened. The median number of contacts screened per household was four (range 1-29; Table 1); 1533 (42.3\%) were males, and the median age was 20 years (range 0- 
TB/HIV contact tracing in South Africa

98), which was lower in males than in females (17 years, range 0-88 vs. 22 years, range 0-98; $\mathrm{P}<0.001$, Mann-Whitney U-test). A history of previous anti-tuberculosis treatment was reported in $219 / 3627$ (6.0\%) contacts. There were respectively 58/3627 (1.6\%) and 144/3627 (4.0\%) contacts currently on anti-tuberculosis treatment and TBPT.

The management of household contacts was different for those aged $<5$ years ( $n=552,15.2 \%$ ) compared to those aged $\geq 5$ years ( $n=3075,84.8 \%$ ), outcomes in these two groups are reported separately and illustrated in the Figure.

\section{Outcomes among contacts aged $<5$ years}

Among 552 contacts aged $<5$ years, 16 (2.9\%) were already on anti-tuberculosis treatment, and an additional 125 (22.6\%) were already taking TBPT and did not have symptoms defining TB suspect status. No contacts in either of these two groups were referred, and they were excluded from further analysis (Figure). Of the remaining 411, 105 (25.5\%) had symptoms defining TB suspect status and were referred for assessment. Among those with known outcomes ( $n=98), 24$ (24.5\%) started anti-tuberculosis treatment and 74 (75.5\%) were commenced on TBPT.

The remaining 306 asymptomatic contacts aged $<5$ years were eligible for referral to potentially start TBPT, and 265 (86.6\%) were referred. Of those referred with a known outcome ( $n=222), 10$ (4.5\%) started anti-tuberculosis treatment and 212 (95.5\%) commenced TBPT (Figure).

Twelve children aged $<5$ years were reported to be HIV-positive by their carer, and an additional $11 / 118$ (9.3\%) tested by the programme were found to be positive. In total, among the 361 household contacts aged $<5$ years seen by the programme with a known outcome, and including those aged $<5$ years assessed and not referred, 34 (9.4\%) were TB cases and 286 (79.2\%) started TBPT (Figure).

\section{Outcomes among contacts aged $\geq 5$ years}

Among 3075 contacts aged $\geq 5$ years, 42 (1.4\%) were already on anti-tuberculosis treatment. These were not further screened for TB, and were excluded from further analysis (Figure). Among the remaining 3033 contacts, 2259 (74.5\%) did not report symptoms defining TB suspect status. Of these, $14(0.6 \%)$ were referred (all age 5-6) to potentially start TBPT, and an additional 25 (1.1\%) were already on TBPT. 
TB/HIV contact tracing in South Africa

The remaining 774 were considered TB suspects based on their symptoms (Figure). The most common symptoms among these suspects were cough $(n=750,96.9 \%)$, sputum production $(n=724$, 93.5\%), weight loss ( $n=112,14.5 \%)$ and night sweats $(n=108,14 \%)$. Using the revised World Health Organization TB symptom screen of cough, fever, weight loss or night sweats of any duration would have identified an additional 16 contacts as TB suspects. ${ }^{9}$ Twenty-five (3.2\%) TB suspects were referred to government medical facilities, as they were considered to require immediate assessment or did not have a sufficiently productive cough. Where outcomes were traced $(n=21), 12(57 \%)$ started anti-tuberculosis treatment, 6 (29\%) started TBPT (all were aged 5) and 3 (14\%) were recorded not to require anti-tuberculosis treatment (Figure).

Of the remaining TB suspects, 727/749 (97.1\%) had sputum collected, with no difference by sex $\left(p=0.636, \chi^{2}\right.$ test). Complete sets of three samples were provided by 662/727 (91.1\%). Among 616 with available results, 71 (11.5\%) were culture-positive for TB of which 9 (12.7\%) were also smearpositive. There were also 10 cases who were smear-positive only. One case was found among eight TB suspects who were already on TBPT. All those who were smear- and/or culture positive were referred to commence anti-tuberculosis treatment. Among those that provided more than one sample, the second smear sample provided the diagnosis in 7/727 (1\%). In total, 93/3029 (3.1\%) started anti-tuberculosis treatment. Their characteristics are described in Table 2.

Among contacts aged $\geq 14$ years, $174 / 2297$ (7.6\%) self-reported as being HIV-positive. Participation in HIV testing among the remainder was $36.9 \%$ (784/2123). Females had a higher uptake, at $41.5 \%$ (531/1281), than males, at $30.0 \%$ (253/842; $\mathrm{P}<0.001, \chi^{2}$ test). In addition, ten contacts aged $\geq 14$ years self-reporting HIV-positive status were retested; a total of 208/794 (26.2\%) tested positive. There were also $17 / 130$ (13.1\%) aged between 5 and under 14 years who tested positive. Including those tested aged $<5$ years, described above, a total of 236/1042 (22.7\%) household contacts tested by programme staff were HIV-positive. Also including those not tested by the programme, but selfreporting positive HIV-status, 424/3627 (11.7\%) household contacts were classified as HIV-positive (reflecting a minimum prevalence, assuming those not tested were HIV-negative).

In univariable analysis of household contacts, risk factors associated with being a TB case were positive HIV status (OR 3.3, 95\% Cl 2.1-5.2) and previous TB (OR 2.6, 95\% Cl 1.4-4.7). In multivariable analysis, HIV infection remained associated with TB case status (OR 3.0, 95\% Cl 1.8-5.1), but previous TB was less strongly associated and of borderline significance (OR 1.8, 95\% Cl 0.95-3.4; Table 2). 


\section{DISCUSSION}

This evaluation illustrates that a household contact based ACF programme can detect otherwise undiagnosed TB and HIV cases in a setting where the burden of both is high. Furthermore, it assists with initiating TBPT in high-risk child contacts to interrupt progression to TB disease.

At $9.4 \%$ among those aged $<5$ years and $3.1 \%$ among those aged $\geq 5$ years, the yield of active TB compares favourably to a recent meta-analysis that reported a median TB yield of $2.2 \%$ (range 0.01 14.5\%) for contact tracing based ACF in resource-limited settings. ${ }^{10}$ Yields from similar recent household ACF studies in African settings were $1.5 \%$ in The Gambia ${ }^{11}, 1.7 \%$ in Malawi ${ }^{12}$ and $6.3 \%$ in Uganda, where four visits were conducted over 24 months $^{13}$. The proportion of children aged $<5$ years treated for active TB through Ribolola was particularly high, which could be because a higher proportion of referrals to clinic were successful compared with other studies. ${ }^{12}$

The programme revealed a high proportion of undiagnosed HIV among household contacts of TB cases. The screening and referral of newly diagnosed HIV cases to clinics is an important benefit of the programme and highlights the advantages of integrated TB and HIV screening and referral for care ${ }^{14}$ Linkage to care for patients with both HIV and TB would be simplified if referral was to a single integrated service, rather than two separate services ${ }^{15}$, particularly in South Africa where over half of incident TB cases also have HIV infection. Earlier diagnosis and treatment of HIV, with treatment of latent TB infection, can reduce the risk of TB disease. ${ }^{16}$ Participation in HIV testing was seemingly low, at 37\%; nonetheless, Ribolola newly identified 236 people with HIV infection. Reasons for declining HIV testing were not collected, but anecdotally include having tested recently and concern regarding confidentiality. Qualitative research could help improve acceptability of home-based HIV testing. Barriers to programmes such as Ribolola include the belief that a significant proportion of TB suspects may not be able to provide sputum at home. ${ }^{17}$ We found that, with education, most individuals could produce sputum, and removing the requirement to attend clinic may help overcome the attrition of contacts.

The second sputum smear was discriminatory in diagnosing TB for only a handful of cases, and has significant cost implications in a large programme. The incremental cost per TB case detected by sequential sputum smears was shown in a Zambian study to increase from US\$4.50 for one smear to US\$23.3 for the second smear (assuming the first had already been performed). ${ }^{18}$

The shortcomings of smear microscopy are known in HIV co-infection, where patients are more likely to be smear-negative. ${ }^{19}$ This could also be the case for those examined by ACF as earlier assessment than would normally be the case could result in a greater likelihood of those contacts 
having paucibacillary sputum. Our data underline the higher sensitivity of sputum culture vs. smear. Newer diagnostic technologies, particularly Xpert MTB/RIF (Cepheid, Sunnyvale, CA, USA), a realtime polymerase chain reaction assay for TB that also detects rifampicin (RMP) resistance in approximately 2 hours, raise prospects for near-to-home TB diagnosis for contacts. The costs are currently high, and while it is very sensitive in smear-positive cases, this sensitivity is reduced in smear-negative cases. ${ }^{20}$ Other limitations include the imperfect specificity of Xpert MTB/RIF for RMP resistance, which is more problematic in a contact tracing setting with low prevalence of drug resistance. ${ }^{21}$

The strengths of this evaluation were that the programme had reliable data spanning nearly 2 years with large enough denominators to provide adequate statistical power for a variety of subgroup analyses. Ribolola reflected programmatic rather than research conditions, and programme guidelines were not always rigorously followed. For instance, some eligible contacts aged $<5$ years were not referred for TBPT assessment, whereas some contacts just over 5 years were referred and started TBPT. This represents a grey area, where clinical judgement and the extent of contact are taken into account, and such flexibility seems appropriate. ${ }^{22} \mathrm{~A}$ potential limitation is that those screened represent household contacts of index cases that gave consent for a visit while in the clinic. This may have led to more engagement with programme services and ease of household location than would have been the case had such contacts been traced and approached without prior consent of the index case.

Although ACF may identify more TB cases, this may not translate into cured cases as historically these patients were more likely to refuse or default on their treatment. ${ }^{23} \mathrm{~TB}$ and HIV treatment outcomes of referred contacts should be examined to determine the population-level impact of the programme. Due to limited resources, the programme did not follow TB cases to determine treatment outcomes, which would otherwise be an important point regarding the value of ACF.

This evaluation suggests under-diagnosis of TB and HIV in high-prevalence settings such as South Africa. Household contacts of index TB cases are at higher risk, and this evaluation illustrates that implementation of household-based ACF programmes can promote identification of active TB and provision of TBPT, particularly for children $<5$ years, who are especially at risk. Given early results from the ZAMSTAR (Zambia/South Africa TB and AIDS Reduction) study showing that household TB and HIV case-finding interventions can reduce TB prevalence at community level, such interventions could contribute to better TB control, and merit higher priority. ${ }^{24}$ 
TB/HIV contact tracing in South Africa

\section{ACKNOWLEDGEMENTS}

The authors thank the Ribolola contact tracing team at the Aurum Institute: S Maboyi, B Makhoana, B Morake, H Mooketsi, C Innes, C Hoffmann and G Gresak. The evaluation of this programme was supported by the Evidence for Action research programme consortium, funded by the United Kingdom Department for International Development. 


\section{REFERENCES}

1. World Health Organization. Global Tuberculosis Control: WHO report. 2011. Available from: http://www.who.int/tb/publications/global_report/en/ [Accessed August 2012]

2. Harries AD, Zachariah R, Corbett EL, Lawn SD, Santos-Filho ET, Chimzizi R, et al. The HIV-associated tuberculosis epidemic--when will we act? Lancet 2010;375(9729):1906-19.

3. De Cock KM, Chaisson RE. Will DOTS do it? A reappraisal of tuberculosis control in countries with high rates of HIV infection. Int J Tuberc Lung Dis 1999;3(6):457-65.

4. Golub JE, Mohan $\mathrm{Cl}$, Comstock GW, Chaisson RE. Active case finding of tuberculosis: historical perspective and future prospects. Int J Tuberc Lung Dis 2005;9(11):1183-203.

5. Claessens NJ, Gausi FF, Meijnen S, Weismuller MM, Salaniponi FM, Harries AD. High frequency of tuberculosis in households of index TB patients. Int J Tuberc Lung Dis 2002;6(3):266-9.

6. Comstock GW, Livesay VT, Woolpert SF. The prognosis of a positive tuberculin reaction in childhood and adolescence. Am J Epidemiol 1974;99(2):131-8.

7. Marais BJ, Gie RP, Schaaf HS, Hesseling AC, Obihara CC, Nelson L, et al. The clinical epidemiology of childhood pulmonary tuberculosis: a critical review of literature from the prechemotherapy era. Int J Tuberc Lung Dis 2004;8(3):278-85.

8. Republic of South Africa Department of Health. South African National Tuberculosis Management Guidelines. 2009. Available from: http://www.tbonline.info/archive/document/5/ [Accessed August 2012]

9. World Health Organization. Guidelines for intensified tuberculosis case-finding and isoniazid preventive therapy for people living with HIV in resource-constrained settings. 2011. Available from: http://www.who.int/hiv/pub/tb/9789241500708/en/index.html [Accessed August 2012]

10. Kranzer K, Houben RM, Glynn JR, Bekker LG, Wood R, Lawn SD. Yield of HIV-associated tuberculosis during intensified case finding in resource-limited settings: a systematic review and meta-analysis. Lancet Infect Dis 2010;10(2):93-102.

11. Jackson-Sillah D, Hill PC, Fox A, Brookes RH, Donkor SA, Lugos MD, et al. Screening for tuberculosis among 2381 household contacts of sputum-smear-positive cases in The Gambia. Trans R Soc Trop Med Hyg 2007;101(6):594-601.

12. Zachariah R, Spielmann MP, Harries AD, Gomani P, Graham SM, Bakali E, et al. Passive versus active tuberculosis case finding and isoniazid preventive therapy among household contacts in a rural district of Malawi. Int J Tuberc Lung Dis 2003;7(11):1033-9.

13. Guwatudde D, Nakakeeto M, Jones-Lopez EC, Maganda A, Chiunda A, Mugerwa RD, et al. Tuberculosis in household contacts of infectious cases in Kampala, Uganda. Am J Epidemiol 2003;158(9):887-98.

14. Abdool Karim SS, Churchyard GJ, Abdool Karim Q, Lawn SD. HIV infection and tuberculosis in South Africa: an urgent need to escalate the public health response. Lancet 2009;374(9693):921-33.

15. Legido-Quigley H, Montgomery CM, Khan P, Atun R, Fakoya A, Getahun H, et al. Integrating tuberculosis and HIV services in low and middle-income countries: a systematic review. Trop Med Int Health [In press 2012].

16. Lawn SD, Badri M, Wood R. Tuberculosis among HIV-infected patients receiving HAART: long term incidence and risk factors in a South African cohort. AIDS 2005;19(18):2109-16.

17. Akhtar S, White F, Hasan R, Rozi S, Younus M, Ahmed F, et al. Hyperendemic pulmonary tuberculosis in peri-urban areas of Karachi, Pakistan. BMC Public Health 2007;7:70.

18. Walker D, McNerney R, Mwembo MK, Foster S, Tihon V, Godfrey-Faussett P. An incremental cost-effectiveness analysis of the first, second and third sputum examination in the diagnosis of pulmonary tuberculosis. Int J Tuberc Lung Dis 2000;4(3):246-51. 
19. Getahun H, Harrington M, O'Brien R, Nunn P. Diagnosis of smear-negative pulmonary tuberculosis in people with HIV infection or AIDS in resource-constrained settings: informing urgent policy changes. Lancet 2007;369(9578):2042-9.

20. Boehme CC, Nicol MP, Nabeta P, Michael JS, Gotuzzo E, Tahirli R, et al. Feasibility, diagnostic accuracy, and effectiveness of decentralised use of the Xpert MTB/RIF test for diagnosis of tuberculosis and multidrug resistance: a multicentre implementation study. Lancet 2011;377(9776):1495-505.

21. Lawn SD, Nicol MP. Xpert(R) MTB/RIF assay: development, evaluation and implementation of a new rapid molecular diagnostic for tuberculosis and rifampicin resistance. Future Microbiol 2011;6(9):1067-82.

22. Hawkridge T. Tuberculosis contacts and prophylaxis. S Afr Med J 2007;97(10 Pt 2):998-1000.

23. Cassels A, Heineman E, LeClerq S, Gurung PK, Rahut CB. Tuberculosis case-finding in Eastern Nepal. Tubercle 1982;63(3):175-85.

24. Ayles H, ZAMSTAR Study Team. A Household-based HIV and TB Intervention Increases HIV Testing in Households and Reduces Prevalence of TB at the Community Level: The ZAMSTAR Community Randomized Trial [abstract \#149bLB]. $19^{\text {th }}$ Conference on Retroviruses and Opportunistic Infections (CROI); 2012 Mar 5-8; Seattle. Available from: http://www.retroconference.org/2012b/Abstracts/45440.htm [Accessed August 2012] 
TB/HIV contact tracing in South Africa

Table 1: Characteristics of screened household contacts $(n=3627)$

\begin{tabular}{|c|c|c|}
\hline \multicolumn{2}{|c|}{ Characteristics } & Number of contacts (\%) \\
\hline \multicolumn{2}{|l|}{ Female } & $2094(57.7 \%)$ \\
\hline \multirow[t]{7}{*}{ Age (years) } & $0-4$ & $552(15.2 \%)$ \\
\hline & $5-14$ & $850(23.4 \%)$ \\
\hline & $15-24$ & $777(21.4 \%)$ \\
\hline & $25-34$ & $511(14.1 \%)$ \\
\hline & $35-44$ & $336(9.3 \%)$ \\
\hline & $45-54$ & $282(7.8 \%)$ \\
\hline & $\geq 55$ & $319(8.8 \%)$ \\
\hline \multicolumn{2}{|c|}{ Tested HIV positive* } & $236(22.7 \%)$ \\
\hline \multicolumn{2}{|c|}{ Self-reported HIV positive status ${ }^{\dagger}$} & $188(7.3 \%)$ \\
\hline \multicolumn{2}{|c|}{$\begin{array}{l}\text { Tested and/or self-reported HIV positive status, of all contacts } \\
(\mathrm{n}=3627 \ddagger\end{array}$} & $424(11.7 \%)$ \\
\hline \multicolumn{3}{|c|}{ TB } \\
\hline \multicolumn{2}{|c|}{ Previously treated for TB } & $219(6.0 \%)$ \\
\hline \multicolumn{2}{|c|}{ Currently on TB treatment } & $58(1.6 \%)$ \\
\hline \multicolumn{2}{|c|}{ Currently taking TB preventive therapy } & $144(4.0 \%)$ \\
\hline \multicolumn{2}{|c|}{$<5$ years } & $125 / 552(22.6 \%)$ \\
\hline \multicolumn{2}{|c|}{$\geq 5$ years } & $19 / 3075(0.6 \%)$ \\
\hline \multicolumn{2}{|c|}{ Born in South Africa $(n=3426)$} & 3289 (96.0\%) \\
\hline \multicolumn{3}{|c|}{ Education status in $\geq 14 s(n=2171)$} \\
\hline \multicolumn{2}{|c|}{ No formal education } & $203(9.4 \%)$ \\
\hline \multicolumn{2}{|c|}{ Grade 1-12 } & $1943(89.5 \%)$ \\
\hline \multicolumn{2}{|c|}{ Higher education, no degree } & $13(0.6 \%)$ \\
\hline \multicolumn{2}{|c|}{ Degree or diploma } & $12(0.6 \%)$ \\
\hline \multicolumn{3}{|c|}{ Occupational status in $\geq 14 s$ ( $n=2169)$} \\
\hline \multicolumn{2}{|c|}{ Unemployed } & $876(40.4 \%)$ \\
\hline \multicolumn{2}{|c|}{ Student } & $573(26.4 \%)$ \\
\hline \multicolumn{2}{|c|}{ Salaried worker } & $429(19.8 \%)$ \\
\hline \multicolumn{2}{|c|}{ Self-employed } & $34(1.6 \%)$ \\
\hline \multicolumn{2}{|c|}{ Occasional work } & $52(2.4 \%)$ \\
\hline \multicolumn{2}{|c|}{ Unable to work } & $59(2.7 \%)$ \\
\hline \multicolumn{2}{|l|}{ Other } & $146(6.7 \%)$ \\
\hline \multicolumn{3}{|c|}{ * Among 1042 tested by the programme } \\
\hline \multicolumn{3}{|c|}{ † Among $n=2585$ not tested by programme } \\
\hline \multicolumn{3}{|c|}{ ‡ Minimum HIV prevalence among contacts } \\
\hline
\end{tabular}


TB/HIV contact tracing in South Africa

Table 2: Characteristics of TB Cases among Household Contacts $\geq 5$ years $(n=3029)$

\begin{tabular}{|c|c|c|c|}
\hline & \multirow[t]{2}{*}{ Cases/Row Total (\%) } & \multicolumn{2}{|c|}{ Odds Ratio (95\% Confidence Interval) } \\
\hline & & Unadjusted & Adjusted* \\
\hline \multicolumn{4}{|l|}{ Sex } \\
\hline Male & $38 / 1250(3.0 \%)$ & 1 & 1 \\
\hline Female & $55 / 1779(3.1 \%)$ & $1.02(0.67-1.55)$ & $0.89(0.58-1.37)$ \\
\hline \multicolumn{4}{|l|}{ Age } \\
\hline $5-14$ & $21 / 840(2.5 \%)$ & $1 \dagger$ & 1 \\
\hline $15-24$ & $16 / 773(2.1 \%)$ & $0.82(0.43-1.59)$ & $0.77(0.40-1.49)$ \\
\hline $25-34$ & $22 / 497(4.4 \%)$ & $1.81(0.98-3.32)$ & $1.25(0.66-2.39)$ \\
\hline $35-44$ & $11 / 328(3.4 \%)$ & $1.35(0.65-2.84)$ & $0.87(0.40-1.90)$ \\
\hline $45-54$ & $11 / 275(4.0 \%)$ & $1.63(0.77-3.41)$ & $1.18(0.55-2.54)$ \\
\hline$\geq 55$ & $12 / 316(3.8 \%)$ & $1.54(0.75-3.17)$ & $1.53(0.74-3.17)$ \\
\hline \multicolumn{4}{|l|}{ HIV } \\
\hline Negative $\ddagger$ & $64 / 2648(2.4 \%)$ & 1 & 1 \\
\hline Positive & $29 / 381(7.6 \%)$ & $3.33(2.12-5.23)$ & $3.04(1.83-5.05)$ \\
\hline \multicolumn{4}{|l|}{ Previous TB } \\
\hline No & $80 / 2840(2.8 \%)$ & 1 & 1 \\
\hline Yes & $13 / 189$ (6.9\%) & $2.55(1.39-4.67)$ & $1.79(0.95-3.39)$ \\
\hline \multicolumn{4}{|c|}{ * Adjusted for sex, age group, HIV, previous TB } \\
\hline \multicolumn{4}{|c|}{+ Non-parametric test for trend $p=0.054$} \\
\hline ‡ Tested nega & rogramme or, self-repc & $\mathrm{kn}$ & \\
\hline
\end{tabular}


TB/HIV contact tracing in South Africa

Figure 1: Flow diagram of outcomes of screened household contacts

Abbreviations:

TBPT - TB preventive therapy

\section{7 screened household contacts}

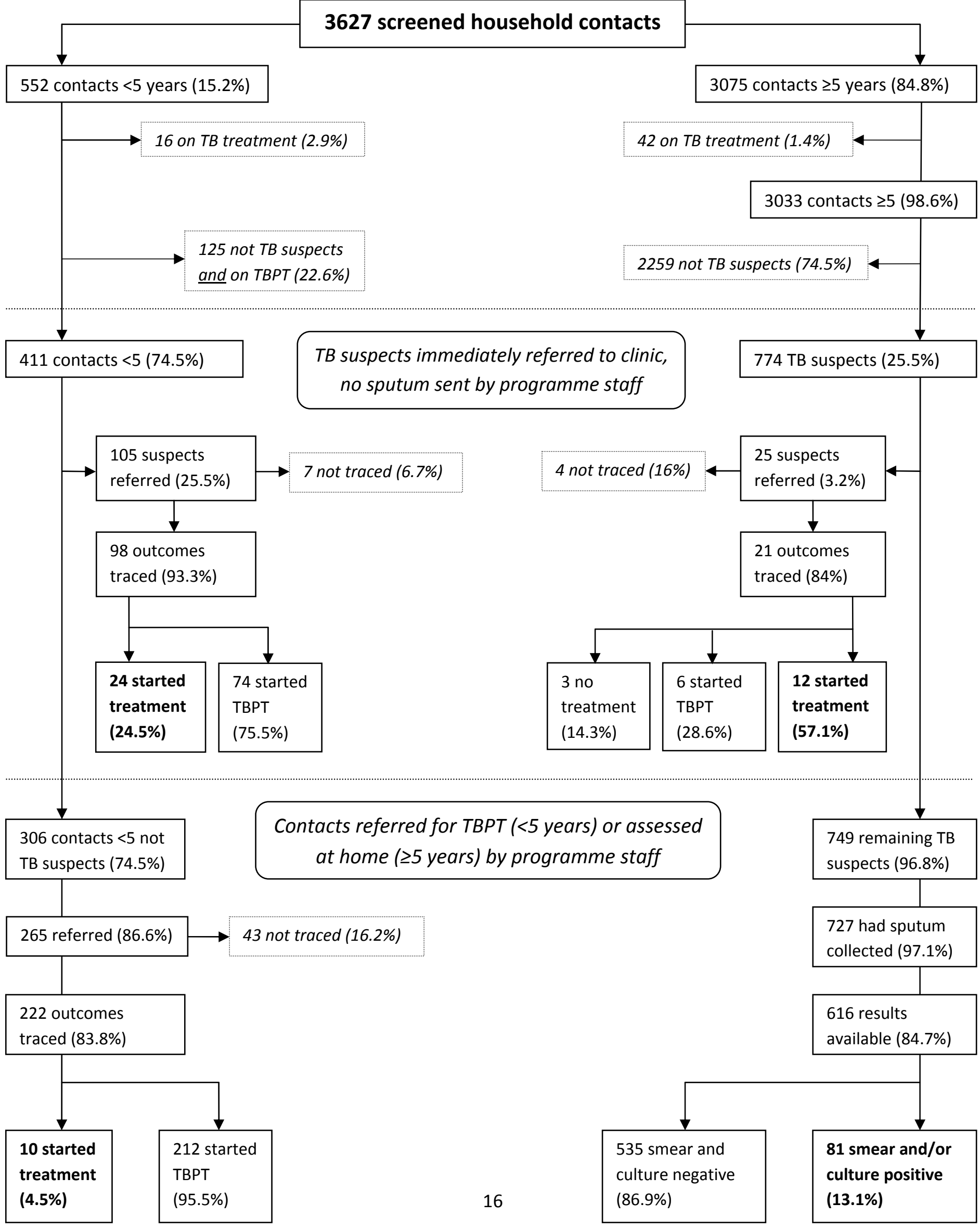

